\title{
LES PRÉPOSITIONS POUR ET CONTRE DANS UNE PERSPECTIVE COMPARATIVE
}

\author{
Iva Dedková
}

\begin{abstract}
In this study, the author analyses the use of the French prepositions pour [for] and contre [against] and examines their reciprocal relation from the semantic viewpoint, presenting the results of the research focused on this issue.

Keywords: pour [for] and contre [against]; prototype; use; near context synonyms; research.

Résumé : Dans cette étude, l'auteur analyse l'usage des prépositions françaises pour et contre et examine leur relation réciproque sur le plan sémantique, en présentant les résultats de l'enquête orientée vers cette problématique.

Mots clés : pour et contre ; prototype ; emploi ; quasi-synonymes occurrentiels ; enquête.
\end{abstract}

\section{Introduction}

Cet article attire l'attention sur les prépositions simples pour et contre et s'interroge en particulier sur leur relation réciproque, avant tout sur le plan sémantique.

Ses différentes parties présentent leurs traits caractéristiques, y compris leurs sens prototypiques et l'éventail de leurs emplois. L'analyse de l'enquête effectuée dans le cadre de cette étude vient compléter la problématique abordée.

Les exemples que nous présentons sont basés sur une variété de sources telles que les articles de presse disponibles en version électronique, les informations fournies sur Internet, les dictionnaires, les monographies et également notre propre expérience.

\section{Sur les caractéristiques générales des termes pour et contre}

Les éléments pour et contre peuvent appartenir à plusieurs catégories morpho-syntaxiques : à côté des emplois prépositionnels, ils connaissent les utilisations substantivée et adverbiale (question terminologique, dans le dernier cas, il est également possible de parler des prépositions à régime implicite). L'emploi conjonctif de pour exige la présence du formème conjonctif que. Voici quelques exemples :

Il est pour cette proposition de loi.

Il y a du pour et du contre.

L'un est pour, l'autre contre.

Nous voulons créer une association pour que la loi change (Midi Libre - Hérault..., 2013). 
Pour et contre entrent dans la composition de nombreux mots et locutions, où ils sont employés en fonction de préfixe, préverbe, adverbe ou préposition (pourboire, pourquoi, par égard pour, contredire, par contre, ci-contre, etc.).

L'étymologie, que nous basons notamment sur l'œuvre de Rey (1992 : 488, 1601-1602), contribue à expliquer les choses.

L'origine de pour est multiple. Il est issu, d'abord sous la forme por et ensuite également pur, du terme latin pro, utilisé en tant que préposition, préverbe et adverbe. Le latin pro s'appliquait aux domaines spatial, temporel et abstrait. En ancien français, les prépositions pour et par ont parfois été confondues, d'où leurs emplois causaux et les alternances possibles. Au cours de l'évolution du français, pour a développé un grand nombre d'usages variés et est devenu l'une des prépositions françaises les plus polysémiques, après de, à, en et dans.

Contre est issu du latin contra, employé comme adverbe et préposition. D'abord, il était orthographié contra, puis cuntre et contre. À partir du XI $\mathrm{I}^{\mathrm{e}}$ siècle, trois sens différents de contre sont déjà affirmés : l'opposition, qui est une notion dominante et par suite prototypique, le contact et la proximité, et également la proportion et la comparaison. D'autres valeurs se développeront ensuite.

La préposition pour appartient aux prépositions françaises les plus fréquentes et également les plus polyvalentes. Elle est multifonctionnelle, ses domaines d'application englobent des emplois spatiaux, temporels et abstraits, les derniers étant très variés.

La préposition contre a une utilisation spatiale et abstraite et marque en particulier, mais non exclusivement, l'opposition.

Selon l'analyse de fréquence des vingt-six prépositions simples du français les «plus typiques » effectuée par Vaguer (2008 : 20-36) sur la base des corpus GEOPO et FRANTEXT, la préposition pour se trouve au $5^{\mathrm{e}}$ rang dans le classement par fréquences croissantes et la préposition contre au $16^{\mathrm{e}}$.

Dans les emplois spatiaux, pour et contre ne sont pas couplés. Dans les domaines $\mathrm{d}^{\prime}$ abstraction, ils peuvent former un couple antonymique $(X)$ ou, par contre, quasi synonymique $^{1}(/)$, ou encore ne pas entrer en couple :

Il est (pour $X$ contre) ce projet.

Les médicaments (pour / contre) le rhume.

Pour est généralement rangé dans le groupe des prépositions "intermédiaires, mixtes » (celles qui ont à la fois un sens concret spatial, mais elles sont appliquées à plusieurs domaines) et contre dans celui des prépositions " colorées, pleines de sens ». ${ }^{2}$ Mais comme l'avance Cadiot (1997: 36), l'assignation d'une préposition à un des trois groupes (c'està-dire vides de sens, pleines de sens et mixtes) est problématique. Lorsque pour et contre indiquent un rapport local, ou encore temporel quant à pour, ils se rapprochent du pôle coloré. Par contre, leurs emplois abstraits sont plus proches du pôle incolore. À propos de pour, Cadiot ${ }^{3}$ avance :

Cette préposition occupe une place paradoxale : elle a une diversité d'usages et de sens qui l'apparente à ces outils vides (ou «incolores ») que sont à et $d e$, mais en même temps, sa signification hors contexte paraît bien tranchée : on peut admettre que c'est celle d'une desti-

\footnotetext{
1 Dans ce cas, on pourrait parler des quasi-synonymes occurrentiels, des prépositions alternantes, des prépositions concurrentes ou des variantes stylistiques.

2 Voir par ex. Cadiot $(1997: 36)$.

3 Cadiot (1997: 15) range pour dans les prépositions « qui sont les plus proches du pôle incolore ».
} 
nation (d'une sorte de cible, éventuellement de trajet), y compris dans les domaines les plus abstraits de l'expérience. Mais, en fonction des contextes, cette valeur simple se monnaie en des effets extrêmement diversifiés. C'est cette tension entre une signification apparemment bien circonscrite et la valeur quasi-illimitée des effets de sens qui fait tout l'intérêt de cette préposition, et c'est à ce titre qu'elle offre un champ particulièrement riche d'enquête (Cadiot 1991 : 14-15).

Et par exemple, Franckel et Paillard (2007 : 7-8) distinguent deux ordres de prépositions : prépositions de «zonage», aussi dites de "division», et prépositions de " discernement ». Ils rangent pour et contre dans le dernier, c'est-à-dire dans les prépositions "qui ne se présentent plus comme "spatialisantes", mais ne sont pas dépourvues pour autant d'une coloration sémantique » (Franckel - Paillard $2007: 8$ ).

Côté prototypie, pour évoque les notions de but et de direction, contre fait songer à l'opposition. Ensuite, pour interpelle contre et vice versa. Finalement, le couple antonymique pour $X$ contre suggère l'accord $X$ le désaccord. Il existe de nombreux exemples du type suivant :

Êtes-vous pour ou contre la peine de mort? (Linternaute.com, 2013).

Dans la traduction, pour et contre évoquent les équivalents tchèques pro et proti ${ }^{4}$ et anglais for et against :

Travailler pour l'UE. - Pracovat pro EU. - Work for the EU.

Les crimes contre l'humanité. - Zločiny proti lidskosti. - Crimes against humanity.

Néanmoins, les équivalents tchèques des deux prépositions sont assez variés : pour peut être traduit par pro, do, na, z, za, proti, kvuili, (na)místo, $k$ ou vưci, et contre par proti, $k, o$, vedle, pred, za ou na. Ajoutons ces quelques exemples:

Il a manqué l'avion pour Bâle. - Zmeškal letadlo do Basileje.

Ils ont réservé la salle pour trois heures. - Sál si rezervovali na tři hodiny.

Il a posé le vélo contre le mur. - Opřel kolo o zed'.

Le médicament (contre / pour) la toux. - Lék (proti kašli / na kašel).

Sa maison est juste contre la mairie (Dubois 1994 : 418). - Jeho di̊m je hned vedle radnice.

On trouve vingt films médiocres contre un bon (Dubois 1994 : 418). - Na jeden dobrý film prípadá dvacet podprüměrných.

Nous reviendrons à la problématique des sens prototypiques également plus loin encore, lors de l'analyse de notre enquête.

Pour peut se combiner avec une autre préposition ou conjonction de subordination :

Idées repas pour après l'école (Vivre délicieusement, 2013).

Quelque chose à grignoter pour pendant la pause.

[...] une laitière avec de l'eau pour si nous avions soif en cours de route [...] $]^{5}$ (Mes souvenirs..., 2013).

Les paires alternantes avec pour ${ }^{6}$ et contre ${ }^{7}$ sont nombreuses et dépendent des valeurs exprimées et des contextes en question. Présentons ces quelques exemples :

4 Pro se construit avec l'accusatif et proti avec le datif.

5 Exemple relevant du français parlé non soutenu.

6 Les concurrents de pour sont très nombreux (en particulier dans le domaine de l'abstraction) : de, à destination de, par, en vue de, dans le but de, en ce qui concerne, au sujet de, afin de, en faveur de, à cause de, en échange de, à la place de, comme, du point de vue, selon, quant à, d'après, aux yeux de, en l'honneur de, par rapport à, envers, à l'égard de, ou même contre, son antonyme prototypique.

7 Voici quelques quasi-synonymes occurrentiels de contre : à côté de, près de, auprès de, sur, avec, contrairement à, moyennant, ou pour, son antonyme prototypique. 
Elle se fait belle (pour le / en vue du) bal.

Ce concert a été donné (pour / en l'honneur de) son anniversaire.

Elle fait jeune (pour / par rapport à) son âge.

Il a agi (contre / contrairement à) nos ordres.

Elle s'est assise (contre / près de / à côté de / auprès de) son ami.

Quant aux propriétés syntaxiques, pour admet une large gamme de compléments : un syntagme nominal, un syntagme prépositionnel, un adverbe, un adjectif, un infinitif, une sous-phrase introduite par que et un complément nul, tandis que contre limite sa combinatoire à deux types de compléments : un syntagme nominal et un complément nul.

Aussi, sur le plan sémantique, pour et contre peuvent être présentés en couple antonymique, ou même quasi synonymique, selon le cas, mais sur le plan syntaxique, leurs propriétés combinatoires diffèrent considérablement.

\section{3. À propos des emplois particuliers des prépositions pour et contre}

\subsection{L'usage spatial de pour}

La préposition pour marque la valeur dynamique et désigne la direction ${ }^{8}$ : Jarmo est parti pour la Finlande.

Dans ce type d'emploi, elle commute, en fonction des contextes, avec d'autres prépositions directionnelles telles que $\grave{a}$, en, sur, vers et avec certaines locutions spécialisées :

Vols (de / pour / vers / sur / à destination de / à l'arrivée de) Paris.

Réservez votre billet de train (pour / vers / à destination de) Bruxelles.

Lorsque pour sert à marquer la notion de direction, il a pour antonymes de, en provenance de, au départ de ou à partir de:

Vols (de / en provenance de / au départ de / à partir de) Paris.

Comme le montre Martinet (sdr., 1979: 184), l'énoncé « Il part pour la France » peut avoir deux lectures, selon le contexte : tantôt il est engagé dans l'armée française (fonction destinative, pour reprendre le terme de Martinet), tantôt il part en direction de la France (fonction spatiale).

Cadiot (1997 : 40) attribue à pour le sens de «trajectoire qui n'atteint pas sa cible».

\subsection{L'usage spatial de contre}

Au sens spatial, la préposition contre exprime les valeurs suivantes : la direction contraire à quelque chose, la proximité immédiate, le contact et la juxtaposition, sans distinguer entre le repos et le mouvement. Ces valeurs peuvent se combiner à la fois, certaines étant présentes, d'autres absentes. Considérons ces quelques exemples :

Pierre nage contre le courant (direction opposée).

L'armoire est contre le mur (l'armoire [la cible] ne doit pas forcément toucher le mur [le site]

$\rightarrow$ simple localisation + proximité immédiate $\rightarrow$ aucun contact, ni opposition, ne doit être présent).

L'échelle s'appuie contre l'arbre (contact + opposition $\rightarrow$ le site sert d'appui, mais non de support ; le dernier étant représenté par la terre).

Un oiseau risque de s'écraser contre une vitre (d'abord direction opposée, puis contact + opposition).

\footnotetext{
8 Cadiot (1991 : 248) attribue à pour spatial les constituants de « direction ou destination ».
} 
Dans certains emplois, contre peut alterner avec une autre préposition :

Pierre a serré Paul (contre / sur) sa poitrine.

La banque est juste (contre / à côté de / près de / auprès de) la mairie.

Cadiot (1997: 40) attribue à contre le sens de « force active résistante ». ${ }^{9}$

\subsection{1 À propos de la relation entre contre et sur}

À propos du trait de contact, celui-ci est notamment caractéristique de la préposition sur. Pourtant, dans le cas des deux prépositions, il peut également s'agir de l'absence de contact. Le contact exprimé par contre diffère de celui marqué par sur. Selon Vandeloise (1986 : 200-205), contre exprime généralement un contact entre deux forces. Les éléments en contact sont mobiles ou immobiles et leur orientation est le plus souvent horizontale ou oblique. Pour ce qui est de sur, l'orientation des éléments en contact est le plus souvent verticale, ou presque verticale. Sur introduit le nom d'un objet qui sert de support (« la position de la cible dépend de celle du site»), mais dans le cas de contre, la cible et le site sont indépendants, entièrement ou partiellement, ou mutuellement dépendants. D'après Martinet (sdr., 1979 : 184), contre ne suppose pas, à la différence de sur, un accrochage à la surface contre laquelle l'objet est placé. À cela ajoutons que, tandis que le site de sur sert de support, le site de contre sert d'appui.

Présentons les exemples suivants :

J'ai mis le canapé contre le mur (contact ou absence de contact).

Pierre s'appuie contre le mur (contact).

Le calendrier est accroché (sur le / au) mur (contact).

Le livre A est contre le livre $B$ (contact $\rightarrow$ les deux livres sont juxtaposés).

Le livre C est sur le livre D (contact $\rightarrow$ le livre C est posé sur le livre D).

\subsection{L'usage temporel de pour}

Au sens temporel, pour indique tantôt un point tempore $\mathrm{l}^{10}$ et répond à " pour quand ? ", tantôt la durée en réponse à « pour combien de temps ? ${ }^{11}$ :

L'envoi sera prêt pour mercredi (point temporel).

Elle va chanter pour une demi-heure (durée).

Ils réservent / Ils ont réservé la salle pour trois heures (ambiguïté : le syntagme pour trois heures peut marquer l'heure de début, ou la durée $\left.{ }^{12}\right)$.

Lorsque pour exprime une durée, il renvoie principalement au présent et au futur :

Elle chante (pour / pendant / durant) une demi-heure.

Elle a chanté (pendant / durant) une demi-heure.

\footnotetext{
9 «Ainsi dans: Max s'est heurté contre le poteau le contact est le résultat d'un mouvement dirigé, d'une force à laquelle l'objet affecté réagit comme une contre-force, ce qui n'est pas du tout le cas dans : Max s'est heurté au poteau. Il en va de même dans des contrastes comme : Max (donne/offre/échange) un briquet (contre + pour) un couteau de poche $[. .] ».($ Cadiot $1997: 258$ - note 57).

10 Šabršula (1989: 153) fait aussi référence à la « localisation temporelle ».

11 Les trois constituants de nature temporelle d'après Cadiot (1991 : 248-249) sont le «terme », le « délai » et la " durée ».

12 Voir aussi Martinet (sdr., $1979: 195)$.
} 


\subsection{L'usage notionnel de pour et de contre}

À part le lieu et le temps, la préposition pour marque une diversité de rapports abstraits. Mentionnons, à titre d'exemple, celles-ci : l'attribution et la favorisation ${ }^{13}$ (La piste noire est trop difficile pour toi.), la cause (Il a reçu une lettre de licenciement pour faute grave [Midi Libre - Alès..., 2013].), la destination et le but (Lait pour le corps à la noix de coco.), l'occasion $^{14}$ (Envoyez vos vœux pour les fêtes de Pâques dans des cartes uniques [Smilebox, 2013].), la conséquence (Je crois qu'il est assez intelligent pour comprendre la complexité de la situation.), le point de vue (Pour moi, elle est la plus belle.), l'opposition (Les médicaments pour maux de gorge.), la concession (Pour une petite fille, elle est très courageuse.), l'échange, le prix, la réciprocité, la comparaison (Abonnez-vous à Midi Libre pour 5,10 €/semaine! [Midi Libre, 2013].), la mise en relief (Pour être jaloux, il l'est.), la proportion (Il y a trois filles pour quatre garçons dans cette classe.), etc.

Pour ce qui est de contre, celui-ci sert à exprimer l'opposition et l'hostilité (Le chat se bat contre le chien.), l'échange (Payez contre ce chèque non endossable $100 €$.) ou la proportion (Il y a trois filles contre quatre garçons dans cette classe.).

Ciblons certains de ces rapports de plus près.

L'opposition peut être marquée non seulement par la préposition contre, mais également par les prépositions avec et pour. Dans le premier exemple ci-dessous, contre commute avec la préposition avec, dans le dernier, il commute avec pour, qui co-désigne aussi la destination, le but :

Le chat se bat (contre/avec) le chien.

Les médicaments (contre/ pour) le rhume.

Nous soutenons l'avis de Cadiot (1997 : 36) qui avance que les prépositions avec ${ }^{15}$ et pour s'interprètent souvent dans ces cas comme « contre ».

Il faut néanmoins ajouter que le syntagme se battre avec a plusieurs lectures différentes possibles, dont «se battre contre » et "se battre du même côté » :

Sans doute l'enfant de 8 ans qui s'enfuit de chez lui pour aller se battre contre les Anglais avec les Boers, [...] (Le général Magrin-Vernerey dit Monclar, 2013).

Le même phénomène peut s'observer en tchèque :

Společně s Angličany a Američany je třeba bojovat proti mad'arské vládě, [...] (Pražský web pro studenou válku, 2013).

Le syntagme se battre pour exprime l'objectif :

Se battre pour une cause juste est déjà une victoire (Publilius Syrus).

Par contre, il existe de très nombreux exemples où pour et contre s'opposent lorsqu'il s'agit du rapport d'opposition. Rappelons cet exemple quasi prototypique :

Êtes-vous pour ou contre la peine de mort?

En ce qui concerne les rapports d'échange et de proportion, dans certains contextes, pour et contre peuvent également se concurrencer :

J'ai échangé une pomme (contre/pour) une poire.

Il y a trois filles (contre / pour) quatre garçons dans cette classe.

13 Terme de Šabršula (1989: 153).

14 Terme de Sandfeld (1965), emprunté par Cadiot (1991 : 249).

15 « [...] où est $l^{\prime}$ ' union > dans être en désaccord avec $x$ ? Se battre avec $x$, c'est le plus souvent se battre contre $x$ ! » (Cadiot $1997: 256$ - note 47). 


\section{4. À propos de l'enquête}

Nous présentons ci-dessous les résultats de l'enquête qui a été effectuée dans la période du 18 au 22 mars 2013 à l'Université de Lorraine à Nancy et qui avait pour groupe cible des Français natifs. 110 personnes y ont participé au total ; il s'agissait avant tout d'étudiants en linguistique française et en didactique du FLE. Les sondés ont été priés de compléter 14 phrases par une préposition ou une locution prépositive. Cette enquête avait pour objectif de relever quelles prépositions semblent naturelles aux locuteurs natifs dans des contextes où, à l'origine, pour et contre étaient utilisés, car différentes prépositions peuvent être employées avec un même contexte-gauche (par ex. un verbe) et un même contexte-droite (groupe nominal). Ceci ne leur a pas été dévoilé avant, bien évidemment.

\begin{tabular}{|c|c|c|}
\hline $\mathrm{N}^{\mathrm{o}}$ & Phrase & Solutions proposées \\
\hline 1. & \begin{tabular}{|l} 
J'ai manqué le train ... Paris. \\
[J'ai manqué le train pour Paris.]
\end{tabular} & pour : 76 // de : 25 // à : 9 \\
\hline 2. & $\begin{array}{l}\text { Ils ont réservé la salle ... trois heures. } \\
\text { [Ils ont réservé la salle pour trois heures.] }\end{array}$ & pour: 78 // à : 19 // pendant : 13 \\
\hline 3. & $\begin{array}{l}\text { Il étudie ... un examen. } \\
\text { [Il étudie pour un examen.] }\end{array}$ & $\begin{array}{l}\text { pour: } \mathbf{1 0 5} / / \text { en vue de }: 3 / / \text { avant: } \\
1 / / 0: 1\end{array}$ \\
\hline 4. & $\begin{array}{l}\text { Le magasin est fermé ... réparations. } \\
\text { [Le magasin est fermé pour réparations.] }\end{array}$ & $\begin{array}{l}\text { pour : } 79 / / \text { pour cause de }: 19 / / \\
\text { à cause de(s) }: 7 / / \text { en raison de }: 3 / / \\
\text { pour cause }: 1 / / \text { pendant les }: 1 \\
\end{array}$ \\
\hline 5. & $\begin{array}{l}\text { Il est grand pour son âge. } \\
\text { [Il est grand ... son âge.] }\end{array}$ & $\begin{array}{l}\text { pour : } 107 / / \text { par rapport à : } 1 / / \text { à : } 1 / / \\
\text { dans : } 1\end{array}$ \\
\hline 6. & $\begin{array}{l}\text { Agent de sécurité chez Cora, il est licencié ... des } \\
\text { croissants non payés. } \\
\text { [Agent de sécurité chez Cora, il est licencié pour des } \\
\text { croissants non payés (Midi Libre - Alès..., 2013).] }\end{array}$ & $\begin{array}{l}\text { pour }: 79 / / \text { à cause de }(\mathrm{s}): 25[\text { de }: 16 / \\
\text { des }: 9] / / \text { en raison de }(\mathrm{s}): 3[\text { de }: 2 / \\
\text { des }: 1] / / \text { pour consommation }(+ \text { de au } \\
\text { lieu de des }): \mathbf{2} / / \text { pour avoir pris }: \mathbf{1}\end{array}$ \\
\hline 7. & $\begin{array}{l}\text { Est-ce que j'aurais risqué ma place ... } 70 \text { centimes ? } \\
\text { [Est-ce que j'aurais risqué ma place pour } 70 \text { centimes ? } \\
\text { (Midi Libre - Alès..., 2013).] }\end{array}$ & $\begin{array}{l}\text { pour : } 106 / / \text { à cause de }: 2 / / \text { contre : } \\
1 / / 0: 1\end{array}$ \\
\hline 8. & $\begin{array}{l}\text { Elles se battent ... Charlotte, Mathieu et Adam. } \\
\text { [(Elles) se battent pour Charlotte, Mathieu et Adam. } \\
\text { (Midi Libre - Hérault..., 2013).] }\end{array}$ & $\begin{array}{l}\text { pour : } 43 / / \text { contre }: 42 / / \text { avec }: 18 / / \\
\text { à cause de }: 5 / / \text { devant }: 1 / / \text { entre }: 1\end{array}$ \\
\hline 9. & $\begin{array}{l}\text {... moi, mon employeur essaye surtout de supprimer } \\
\text { des postes d'agents de sécurité afin de sous-traiter } \\
\text { cette activité. } \\
\text { [Pour moi, mon employeur essaye surtout de supprimer } \\
\text { des postes d'agents de sécurité afin de sous-traiter cette } \\
\text { activité. (Midi Libre - Alès..., 2013).] }\end{array}$ & $\begin{array}{l}\text { pour : } 65 \text { // selon : } 34 \text { // d'après : } 10 / / \\
0: 1\end{array}$ \\
\hline 10. & $\begin{array}{l}\text { Tous les hommes sont égaux ; il n'y a pas de } \\
\text { discrimination ... les Noirs. } \\
\text { [Tous les hommes sont égaux; ; n'y a pas de } \\
\text { discrimination pour les Noirs (Cadiot } 1991: 230) .]\end{array}$ & $\begin{array}{l}\text { envers }: 51 / / \text { contre }: \mathbf{3 6} / / \text { pour }: \mathbf{1 0} / / \\
\text { avec }: 7 / / \text { entre }: 2 / / \text { vers }: 1 / / \text { chez }: \\
1 / / \text { vis-à-vis }: 1 / / 0: 1\end{array}$ \\
\hline 11. & $\begin{array}{l}\text { Sa maison est juste ... la mairie. } \\
\text { [Sa maison est juste contre la mairie (Dubois } 1994 \text { : } \\
\text { 418).] }\end{array}$ & $\begin{array}{l}\text { à côté de }: 36 / / \text { devant }: 24 / / \text { contre }: \\
14 / / \text { à côté }: 8 / / \text { derrière }: 7 / / \text { près } \\
\text { de }: 4 / / \text { en face de }: 4 / / \text { après }: 3 / / \\
\text { pour }: 3 / / \text { à deux pas de }: 2 / / \text { avant }: \\
2 / / \text { en face }: 1 / / \text { vers }: 1 / / \text { à }: 1\end{array}$ \\
\hline
\end{tabular}




\begin{tabular}{|c|c|c|}
\hline 12. & $\begin{array}{l}\text { Les médicaments ... la toux. } \\
\text { [Les médicaments (contre/pour) la toux.] }\end{array}$ & $\begin{array}{l}\text { contre : } 78 / / \text { pour : } 28 / / \text { [contre / } \\
\text { pour] }: 4\end{array}$ \\
\hline 13. & $\begin{array}{l}\text { On trouve vingt films médiocres ... un bon. } \\
\text { [On trouve vingt films médiocres contre un bon (Dubois } \\
1994: 418) .]\end{array}$ & $\begin{array}{l}\text { pour }: 56 / \text { contre }: 21 / / \text { et }: 20 / / \\
0: 4 / / \text { sur }: 2 / / \text { dont }: 2 / / \text { avant }: 1 / / \\
\text { dans }: 1 / / \text { avec }: 1 / / \text { et seulement }: 1 / / \\
\text { donc }: 1\end{array}$ \\
\hline 14. & $\begin{array}{l}\text { Hérault : trois mamans en guerre ... les tueurs de la } \\
\text { route. } \\
\text { [Hérault : trois mamans en guerre contre les tueurs de la } \\
\text { route (Midi Libre - Hérault..., 2013).] }\end{array}$ & $\begin{array}{l}\text { contre }: 97 / / 0: 4 / / \text { pour }: 3 / / \text { avec }: \\
2 / / \text { et }: 2 / / \text { envers }: 1 / / \text { vers }: 1\end{array}$ \\
\hline
\end{tabular}

Considérons les résultats obtenus.

Phrase $\mathrm{n}^{\circ} 1$ : La majorité des sondés, 76 sur 110, a employé la préposition spatiale directionnelle pour. 25 sondés ont opté pour la préposition de, dont l'emploi est ambigu, car elle ne précise pas s'il s'agit d'un train en provenance ou à destination de Paris. 9 sondés ont utilisé la préposition spatiale $a ̀$, qui n'est pas un synonyme occurrentiel des prépositions pour et $d e$.

Phrase $\mathrm{n}^{\circ} 2: 78$ sondés sur 110 ont utilisé la préposition temporelle pour, les autres ont employé à (19) ou pendant (13). Chacune de ces trois prépositions a un impact considérable sur le sens de la phrase - pour indique l'heure de début ou la durée, à l'heure précise où la réservation a été effectuée et pendant la durée.

Phrase $n^{\circ} 3$ : La plupart des sondés, précisément 105 , ont eu recours à la préposition notionnelle de but pour. 3 sondés se sont servis de son quasi-synonyme occurrentiel en vue de. La préposition avant, usée par 1 seul sondé, combine dans ce contexte une valeur temporelle et une valeur notionnelle de but.

Phrase $\mathrm{n}^{\circ} 4$ : Les sondés ont complété cette phrase par une variété de prépositions marquant la cause, avec au premier rang pour (79), suivi de ses concurrents : pour cause de (19), à cause de (7), en raison de (3) et pour cause (1). 1 seul sondé a souligné l'aspect temporel en utilisant la préposition durative pendant.

Phrase $\mathrm{n}^{\circ} 5$ : Dans leur très grande majorité, les sondés ont fait usage de la préposition pour (107 emplois), qui marque ici la comparaison. Leurs quasi-synonymes occurrentiels par rapport à et à n'ont été utilisés que par 1 seul sondé chaque fois. Nous trouvons l'utilisation de la préposition dans (1 sondé) dans cette phrase non pertinente.

Phrase $\mathrm{n}^{\circ} 6$ : La plupart des sondés, 82 (ou $79+2+1$ ), se sont servis de la préposition pour, dont 2 l'ont renforcé par consommation et 1 par avoir pris. Les autres ont utilisé, pour marquer la cause, ses quasi-synonymes occurrentiels à cause de (25 sondés) et en raison de (3), dont le premier implique un résultat négatif et le dernier un résultat neutre.

Phrase $\mathrm{n}^{\circ} 7$ : Les sondés ont majoritairement eu recours à la préposition pour (106 emplois), qui sert à indiquer la notion d'échange. 1 sondé a utilisé son concurrent contre et 2 sondés ont employé la locution à cause de.

Phrase $n^{\circ} 8$ : Le contexte de cette phrase, en liaison avec le verbe se battre, implique plusieurs valeurs différentes, comme il en découle des résultats de l'enquête : notamment l'opposition, le but et la cause, mais aussi celle de « en présence de ». Au total, 42 sondés ont opté pour la notion d'opposition en employant la préposition contre. 18 sondés se sont servis de la préposition avec : celle-ci évoque l'accompagnement et son utilisation dans ce type de phrase a un double sens, car tantôt elle garde son sens prototypique de l'accompagnement, tantôt elle signifie « contre » et son emploi peut paraître " théo- 
riquement bizarre », mais pourtant assez fréquent. Par contre, 43 sondés ont eu recours à la notion de but par l'intermédiaire de la préposition pour, qui a été originellement employée dans la phrase citée. 5 sondés se sont servis de la locution à cause de. 1 sondé a utilisé la préposition devant pour dire " en présence de » et 1 sondé a employé entre.

Phrase $n^{\circ}$ 9: 65 sondés ont utilisé pour, 34 selon et 10 d'après. Le syntagme prépositionnel pour moi peut avoir un double sens : tantôt il sert à mettre en relief le thème de l'énoncé et signifie «en ce qui me concerne » ou "quant à moi ", tantôt il introduit le point de vue et a pour variante stylistique « à mes yeux ». Selon moi et d'après moi peuvent être interprétés comme «je le pense ».

Phrase $\mathrm{n}^{\circ} 10$ : Les sondés ont utilisé une variété de prépositions, dont les suivantes servent à marquer l'attitude : envers (51), contre (36), pour (10), avec (7), vers (1) et vis-àvis (1). Les syntagmes entre les Noirs et chez les Noirs changent le sens initial de la phrase pour « tous les Noirs sont égaux » - entre (2) marque la réciprocité, chez (1) la présence ou l'absence de quelque chose dans le comportement de quelqu'un.

Phrase $n^{\circ} 11$ : Le contexte de cette phrase peut être considéré «assez libre», car il implique simplement l'emploi d'une préposition locative statique. C'est pourquoi les sondés ont eu recours à une grande variété de prépositions spatiales : à côté de (36), devant (24), contre (14), à côté (8), derrière (7), près de (4), en face de (4), à deux pas de (2), en face (1), vers (1), à (1), et aux prépositions spatio-temporelles antonymiques après (3) et avant (2). Notons que les locutions à côté de, près de et à deux pas de (de même que auprès de) peuvent alterner avec la préposition contre, originellement utilisée dans cette phrase, pour marquer la proximité immédiate dans l'espace. Par contre, avec la préposition pour (3), on sort du domaine spatial, car le sens originel de la phrase change pour «sa maison soutient justement la mairie ».

Phrase $\mathrm{n}^{\circ} 12$ : Le contexte de la phrase n'admet que l'emploi de deux prépositions, voir contre et pour, qui servent à désigner l'opposition. Voilà l'exemple où pour et contre cessent d'être antonymes pour devenir des quasi-synonymes occurrentiels. 78 sondés ont donc utilisé contre, 28 sondés ont employé pour et 4 sondés ont tenu à compléter les deux prépositions à la fois [contre / pour].

Phrase $\mathrm{n}^{\circ} 13: 79$ sondés sur 110 se sont servis d'une préposition marquant le rapport de proportion : pour (56), contre (21) et sur (2) ; néanmoins, l'emploi de sur n'est pas pragmatiquement valide. Les prépositions avant, dans et avec ont été employées chacune une seule fois ; l'emploi de dans et peut-être aussi celui de avant nous paraît bizarre. 24 sondés ont eu recours, malgré la consigne, à un emploi non-prépositionnel : et (20), dont (2), et seulement (1) et donc (1).

Phrase $\mathrm{n}^{\circ} 14$ : Dans ce cas, les sondés ont majoritairement fait usage des prépositions qui expriment l'opposition, voir contre, employé 97 fois, et son concurrent avec, usité seulement 2 fois. 3 sondés se sont servis de la préposition pour indiquant le rapport de cause. Les prépositions envers et vers se sont vues employées 1 seule fois chacune. 2 sondés ont utilisé la conjonction et, malgré la consigne.

Ensuite, après avoir remis les questionnaires, il a été demandé à une moitié des sondés de noter ce qu'évoquent pour eux les prépositions pour et contre. Comme il s'agissait de questions ouvertes, le nombre de réponses n'était pas limité.

Aux yeux de nos sondés, la préposition pour évoque notamment :

i. le but (22 mentions),

ii. une connotation positive (9 mentions), 
iii. la cause (8 mentions),

iv. l'accord (6 mentions),

v. être en faveur de quelque chose ou quelqu'un (6 mentions),

vi. la destination (5 mentions),

vii. l'avis : selon (5 mentions),

viii. le partenariat, l'amitié, l'union, le regroupement et la coopération (5 mentions),

ix. la destination géographique et la direction (4 mentions),

x. le temps : la durée (3 mentions).

Pour nos sondés, la préposition contre évoque en particulier :

i. l'opposition (32 mentions) et le contraste (3 mentions),

ii. une connotation négative (12 mentions),

iii. le désaccord ( 9 mentions),

iv. le lieu (8 mentions),

v. la proximité ( 5 mentions),

vi. l'image de combat (5 mentions).

\section{Conclusion}

La préposition pour est considérablement plus fréquente et plus polysémique que contre, qui se situe au contraire plus proche du pôle coloré. Pour a des emplois spatiaux, temporels et notionnels, contrairement à contre qui ne s'applique pas au temps. Vu leur relation réciproque sur le plan sémantique, nous pouvons distinguer trois cas de figure : i. pour et contre forment un couple d'antonymes (il ne s'agit pas d'antonymes réciproques), ii. pour et contre forment un couple de quasi-synonymes occurrentiels (il ne s'agit pas de synonymes absolus), iii. pour et contre ne sont pas couplés (ils ne s'opposent ni commutent). Pour spatial et contre spatial sont des prépositions non antonymiques, ou encore non synonymiques. Pour et contre forment un couple d'antonymes ou de quasi-synonymes d'ordre notionnel. D'après les résultats de notre enquête, les deux prépositions évoquent pour les locuteurs natifs plusieurs notions, dont avant tout le but (pour) et l'opposition (contre). L'enquête a dévoilé quelles prépositions semblent naturelles aux locuteurs natifs dans des contextes où, à l'origine, pour et contre étaient utilisés. Elle a également mis en relief certains contextes où pour et contre commutent avec une multitude d'autres prépositions.

\section{Bibliographie}

CADIOT, Pierre (1991), De la grammaire à la cognition: la préposition pour, Paris : Éditions du CNRS.

CaDiot, Pierre (1997), Les Prépositions abstraites en français, Paris : Armand Colin/ Masson.

Dubois, Jean (sdr., 1994), Dictionnaire de la langue française, Paris : Larousse.

FrancKel, Jean-Jacques - PAILlard, Denis (2007), Grammaire des prépositions, Paris : Éditions Ophrys.

Martinet, André (sdr., 1979), Grammaire fonctionnelle du français, Paris : Didier.

REY, Alain (1992), Dictionnaire historique de la langue française, Paris : Le Robert.

ŠABRŠUla, Jan (1989), Les espèces de relation - Nové kapitoly z rozboru moderní francouzštiny IV, Praha : Univerzita Karlova v Praze, SPN.

VAGuer, Céline (2008), «Classement syntaxique des prépositions simples du 
Les prépositions pour et contre dans une perspective comparative

français ", in : LEEMAN, Danielle (éd.), Langue française $n^{\circ} 157$ : Énigmatiques prépositions, Paris : Larousse, 20-36.

VANDELOISE, Claude (1986), L'espace en français : sémantique des prépositions spatiales, Paris : Éditions du Seuil.

\title{
Sitographie
}

Le général Magrin-Vernerey dit Monclar, http://www.honneurshereditaires.net/ AHH_1_1_4.htm [en ligne le 28/04/2013].

Linternaute.com - Êtes-vous pour ou contre la peine de mort ?, http:/ / www.linternaute. $\mathrm{com} /$ savoir/temoignage/appelatemoin/2131/etes-vous-pour-ou-contre-lapeine-de-mort/ [en ligne le 06/04/2013].

Mes souvenirs..., http:/ / lacorneille.vefblog.net/2.html [en ligne le 08/05/2013].

Midi Libre, http:/ / www.midilibre.fr/2013/03/12/montpellier-la-voiture-finit-surle-toit-au-chu,658544.php [en ligne le 12/03/2013].

Midi Libre - Alès : agent de sécurité chez Cora, il est licencié pour des croissants non payés, http://www.midilibre.fr/2013/01/30/licencie-pour-des-croissants-nonpayes,635727.php [en ligne le 12/03/2013].

Midi Libre - Hérault: trois mamans en guerre contre les tueurs de la route, http:/ / www. midilibre.fr/2013/03/12/ herault-trois-mamans-en-guerre-contre-les-tueursde-la-route,658598.php [en ligne le 12/03/2013].

Pražský web pro studenou válku - Výslech Lazara Brankova, http://www. praguecoldwar.cz/rajkproces4.htm [en ligne le 28/04/2013].

Smilebox - Envoyez vos vœux de Pâques dans des designs uniques !, http://www. smilebox.com/fr/paques/ [en ligne le 06/04/2013].

Vivre délicieusement, https://lifemadedelicious.ca/fr-CA/Recipes/Top10/ AfterSchoolDinnerSpecials.aspx [en ligne le 06/04/2013].

\author{
Iva Dedková \\ Katedra romanistiky \\ Filozofická fakulta \\ Ostravská univerzita v Ostravě \\ Reální 5 \\ 70103 Ostrava \\ République tchèque \\ iva.dedkova@osu.cz
}

